\title{
Entanglement versus Gaussian quantum discord in a double-cavity opto-mechanical system
}

\author{
J. El Qars ${ }^{d}$, M. Daoud ${ }^{b, c, d 2}$ and Ahl Laamara ${ }^{a, e} 3$ \\ ${ }^{a}$ LPHE-MS, Faculty of Sciences, University Mohammed V, Rabat, Morocco \\ ${ }^{b}$ Max Planck Institute for the Physics of Complex Systems, Dresden, Germany \\ ${ }^{c}$ Abdus Salam International Centre for Theoretical Physics, Miramare, Trieste, Italy \\ ${ }^{d}$ Department of Physics, Faculty of Sciences, University Ibnou Zohr, Agadir, Morocco \\ ${ }^{e}$ Centre of Physics and Mathematics (CPM), University Mohammed V, Rabat, Morocco
}

\begin{abstract}
In this paper we investigate the robustness of the quantum correlations against the environment effects in various opto-mechanical bipartite systems. For two spatially separated opto-mechanical cavities, we give analytical formula for the global covariance matrix involving two mechanical modes and two optical modes. The logarithmic negativity as an indicator of the degree of entanglement and the Gaussian quantum discord which is a witness of quantumness of correlations are used as quantifiers to evaluate the different pairwise quantum correlations in the whole system. The evolution of the quantum correlations existing in this opto-mechanical system are analyzed in terms of the thermal bath temperature, squeezing parameter and the opto-mechanical cooperativity. We find that with desirable choice of these parameters, it is possible either enhance or annihilate the quantum correlations in the system. Various scenarios are discussed in detail.
\end{abstract}

\footnotetext{
${ }^{1}$ email: j.elqars@gmail.com

${ }^{2}$ email: m_daoud@hotmail.com

${ }^{3}$ email: ahllaamara@gmail.com
} 


\section{Introduction}

Quantum correlations transfer between light and matter is currently viewed as a key ingredient for future applications in the context of quantum communications and information processing [1, 2, 3, 4]. Storing information in the matter degree of freedom is preferable to overcome the difficulties of storage and localization encountered with photons. In this context, over the last two decades, the transfer of quantum correlations from photons to matter has raised widespread interest from a purely theoretical point of view supported by significant experimental achievements. In fact, the opto-mechanical coupling between the electromagnetic mode in a quantum cavity and the mechanical motion of a nano-mechanical resonator by exploiting the radiation pressure force offers a platform to explore the entanglement transfer between light and matter. The opto-mechanical systems provide also very promising tools to create and manipulate entanglement at mesoscopic scales. The appropriate setup extensively used in investigating quantum correlations in opto-mechanical systems, and subsequently to understand the entanglement transfer between optical and mechanical modes, is the Fabry-Perot cavity [5, 6. Indeed, various schemes using Fabry-Perot cavity were reported in the literature from several perspectives and for different purposes [7, 8, 9, 10, 11, 12, 13, 14, 15, 16, 17. Clearly, the increasing interest in transferring the quantum correlations from microscopic systems to mesoscopic ones is primarily motivated by the use of non-classical entangled states of continuous variable systems quantum information processing, communication and computation. Different measures to quantify the degree of intricacy in bipartite quantum systems were discussed in the literature. In particular, for a long, time the entanglement [18] has been regarded as the key ingredient to distinguish between entangled and separable states and subsequently between the quantum and classical correlations. In this picture, separability has been often identified with the absence of quantum correlations. However, now it is well established that quantum correlation can be present in separable states. Indeed, the notion of quantum discord, introduced in [19, 20], which goes beyond the entanglement, is the appropriate measure to deal with the quantum correlations in bipartite quantum systems, especially the ones those prepared in mixed states. The quantum discord, originally defined and evaluated for finite dimensional system, was extended to the domain of continuous variable systems and especially in analyzing the bipartite quantum correlations in Gaussian mode states [21, 22, 23].

In this paper, to quantify the degree of quantum correlations, we shall use the logarithmic negativity and the Gaussian quantum discord. We stress that the characterization of quantum correlations in opto-mechanical systems is essential to understand the transfer of (quantum correlations from light to matter) entanglement between optical and macroscopic vibrational modes. We notice that other measures and criteria were used in this sense. One may quote for instance, Duan and Simon entanglement criterion proposed simultaneously and independently by Duan et al [24] and Simon [25] which provide the inseparability condition of two continuous variable systems, the Mancini separability criterion [7] which is valid for any state of any bipartite system and generalizes the already mentioned criteria. The logarithmic negativity [26, 27] was also used to quantify the amount between two Gaussian modes. 
However, this measure is not sufficient to specify completely the quantum correlations present in the system, especially for mixed states. Henceforth, the appropriate measure in this case is the Gaussian quantum discord [22] ( see also [21]). In fact, this measure has been shown useful in determining the non-classical correlations between two spatially distant mechanical oscillators [28]. In this work, we investigate the non-classical correlations between the different modes in an opto-mechanical system consisting of two movables mirrors of two spatially separated Fabry-Perot cavities. Each cavity is pumped by a squeezed light. A complete description of this opto-mechanical model is provided in section II. We give the corresponding Hamiltonian. We solve the associated quantum Langevin equations to determine the explicit form of the global covariance matrix involving the quadratures of mechanical and optical modes. In section III, using the logarithmic negativity, we investigate the separability between the different modes in model. A special emphasis is devoted in section IV to the situation where the logarithmic negativity is zero. In this case, the Gaussian quantum discord is used to quantify the quantum correlations appear beyond entanglement. Concluding remarks close this paper.

\section{System and Hamiltonian}

\subsection{The system}

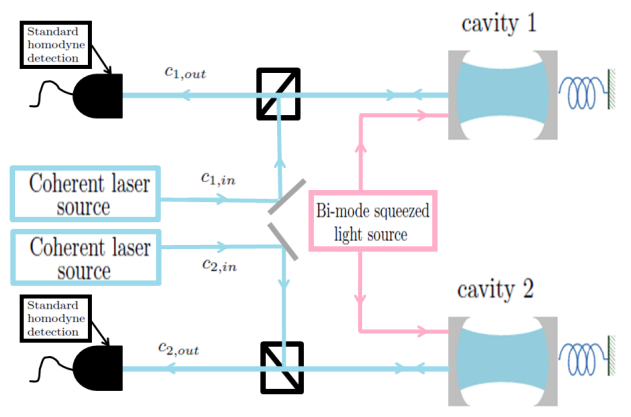

Figure 1: Schematic of two identical opto-mechanical Fabry-Perot cavities which are pumped by identical laser fields (power P and frequency $\omega_{L}$ ) and two-mode squeezed light, generated for example by the spontaneous parametric down conversion source (SPDC). Each movable mirror is treated as a mechanical oscillator characterized respectively by the frequency $\omega_{\mu}$ and the damping rate $\gamma$.

The opto-mechanical system considered in this paper, consists of two identical Fabry-Perot cavities (see Fig.1). Each cavity is composed by two mirrors. The first mirror is fixed and partially transmitting, the second is movable and perfectly reflecting. As depicted in Fig.1, each cavity is pumped simultaneously by coherent laser field and squeezed light produced by using either the SPDC source (spontaneous parametric down-conversion) [29, 30] or by techniques of nonlinear optic [31, 32].

$\varepsilon=\sqrt{\frac{2 \kappa P}{\hbar \omega_{L}}}$ is the amplitude of the pump laser, where the parameter $\kappa$ denotes the energy decay rate of the two cavities, $\omega_{L}$ and $P$ are respectively the frequency and the power of the external laser sources. The opto-mechanical coupling via the radiation pressure [33] between the cavity field and the movable 
mirror is characterized by the coefficient $g$ given by $g=\frac{\omega_{c}}{L} \sqrt{\frac{\hbar}{\mu \omega_{\mu}}}$ with $\omega_{c}$ and $L$ denoting respectively the frequency and the length of each cavity. Finally, each movable mirror will be treated as a quantum mechanical harmonic oscillator with the damping rate $\gamma$, the mass $\mu$ and the frequency $\omega_{\mu}$.

\subsection{The Hamiltonian}

In the in a frame rotating with $\omega_{L}$, the Hamiltonian of the system is given by 34

$$
H=\sum_{i=1}^{2}\left(\left(\omega_{c}-\omega_{L}\right) c_{i}^{\dagger} c_{i}+\omega_{\mu} b_{i}^{\dagger} b_{i}+g c_{i}^{\dagger} c_{i}\left(b_{i}^{\dagger}+b_{i}\right)+\varepsilon\left(e^{i \varphi_{i}} c_{i}^{\dagger}+e^{-i \varphi_{i}} c_{i}\right)\right),
$$

where $c_{i}^{\dagger}$ and $c_{i}$ are respectively the creation and the annihilation operators for the $i^{\text {th }}$ optical mode. They satisfy the usual bosonic commutation relations. Similarly, $b_{i}^{\dagger}$ and $b_{i}$ stand for the creation and the annihilation operators for the $i^{\text {th }}$ mechanical mode. In Eq. (11), $\varphi_{i}$ denotes the $i^{t h}$ input laser field phase. To simplify, we assume $\varphi_{1}=\varphi_{2}=\varphi$. In the Heisenberg representation, the quantum Langevin equations for optical and mechanical modes read

$$
\begin{aligned}
& \frac{d c_{i}}{d t}=i\left[H, c_{i}\right]-\frac{\kappa}{2} c_{i}+\sqrt{\kappa} c_{i}^{i n}=-\left(\frac{\kappa}{2}-i \Delta\right) c_{i}-i g c_{i}\left(b_{i}^{\dagger}+b_{i}\right)-i \varepsilon e^{i \varphi}+\sqrt{\kappa} c_{i}^{i n}, \\
& \frac{d b_{i}}{d t}=i\left[H, b_{i}\right]-\frac{\gamma}{2} b_{i}+\sqrt{\gamma} \xi_{i}=-\left(\frac{\gamma}{2}+i \omega_{\mu}\right) b_{i}-i g c_{i}^{\dagger} c_{i}+\sqrt{\gamma} \xi_{i},
\end{aligned}
$$

where $\Delta=\omega_{L}-\omega_{c}$ is the laser detuning, $c_{i}^{i n}$ denotes the $i^{\text {th }}$ input squeezed vacuum noise operator, $\xi_{i}$ is the $i^{\text {th }}$ noise operator associated to the Brownian motion of the $i^{\text {th }}$ movable mirror. The input squeezed vacuum noise operators $c_{i}^{i n}$ have the following nonzero frequency-domain correlation functions [35, 36]

$$
\begin{aligned}
\left\langle c_{i}^{i n^{\dagger}}(-\omega) c_{i}^{i n}\left(\omega^{\prime}\right)\right\rangle & =2 \pi N \delta\left(\omega+\omega^{\prime}\right), \\
\left\langle c_{i}^{i n}(\omega) c_{i}^{i n^{\dagger}}\left(-\omega^{\prime}\right)\right\rangle & =2 \pi(N+1) \delta\left(\omega+\omega^{\prime}\right), \\
\left\langle c_{1}^{i n}(\omega) c_{2}^{i n}\left(\omega^{\prime}\right)\right\rangle & =2 \pi M \delta\left(\omega+\omega^{\prime}-2 \omega_{\mu}\right), \\
\left\langle c_{1}^{i n^{\dagger}}(-\omega) c_{2}^{i n^{\dagger}}\left(-\omega^{\prime}\right)\right\rangle & =2 \pi M \delta\left(\omega+\omega^{\prime}+2 \omega_{\mu}\right),
\end{aligned}
$$

with $N=\sinh ^{2} r$ and $M=\sinh r \cosh r$, where $r$ is the squeezing parameter characterizing the squeezed light. The noise operators $\xi_{i}$ in Eq. (3) have zero mean value. In general, the mechanical baths are not Markovian [10, 37]. The mechanical baths can be considered as Markovian when the mechanical oscillator frequency $\omega_{\mu}$ is larger than the damping rate $\gamma$. In this situation, we have the following Markovian delta-correlated relations

$$
\begin{aligned}
& \left\langle\xi_{i}^{\dagger}(-\omega) \xi_{i}\left(\omega^{\prime}\right)\right\rangle=2 \pi n_{\mathrm{th}} \delta\left(\omega+\omega^{\prime}\right), \\
& \left\langle\xi_{i}(\omega) \xi_{i}^{\dagger}\left(-\omega^{\prime}\right)\right\rangle=2 \pi\left(n_{\mathrm{th}}+1\right) \delta\left(\omega+\omega^{\prime}\right),
\end{aligned}
$$

where $n_{\text {th }}=\left(\exp \left[\frac{\hbar \omega_{\mu}}{K_{B} T}\right]-1\right)^{-1}$ is the mean thermal photons number and $T$ is the mechanical bath temperature. The quadratic terms in Eqs. (2) and (3) are due essentially to the non-linear nature of the radiation pressure [28]. To solve the system of Eqs. (21) and (3), we define the operators [38]

$$
\delta b_{i}=b_{i}-b_{s i}, \quad \delta c_{i}=c_{i}-c_{s i}
$$


where $b_{s i}$ and $c_{s i}$ are the steady-state averages for mechanical and optical operators respectively. From Eqs. (2) and (3), one can check that they are given by

$$
\left\langle c_{i}\right\rangle=c_{s i}=\frac{-i \varepsilon e^{i \varphi}}{\frac{\kappa}{2}-i \Delta_{\mathrm{eff}}} \quad\left\langle b_{i}\right\rangle=b_{s i}=\frac{-i g\left|c_{s i}\right|^{2}}{\frac{\gamma}{2}+i \omega_{\mu}},
$$

with $\Delta_{\text {eff }}=\Delta-g\left(b_{s i}+\bar{b}_{s i}\right)$ denotes the effective cavity detuning including the mirrors displacements due to radiation pressure. Reporting Eq. (10) in Eqs. (21) and (3), the fluctuations $\delta b_{i}$ and $\delta c_{i}$ of the operators $c_{i}$ and $b_{i}$, around the steady states, obey to the following equations

$$
\begin{aligned}
\delta \dot{c}_{i} & =-\left(\frac{\kappa}{2}-i \Delta_{\mathrm{eff}}\right) \delta c_{i}-G\left(\delta b_{i}^{\dagger}+\delta b_{i}\right)+\sqrt{\kappa} c_{i}^{i n}, \\
\delta \dot{b}_{i} & =-\left(\frac{\gamma}{2}+i \omega_{\mu}\right) \delta b_{i}+G\left(\delta c_{i}-\delta c_{i}^{\dagger}\right)+\sqrt{\gamma} \xi_{i},
\end{aligned}
$$

with $G=g\left|c_{s i}\right|$ is the many-photon opto-mechanical coupling. In deriving the last evolution equations, we have deliberately chosen the input field phase to be $\tan \varphi=\frac{-2 \Delta_{\text {eff }}}{\kappa}$. This is legitimate since the coherent field can be produced with an arbitrary phase. For this special value of the phase, we have $c_{s i}=-i\left|c_{s i}\right|$. Furthermore, setting $\Delta_{\text {eff }}=-\omega_{\mu}$, which corresponds to the quantum state transfer [2]. Using the rotating wave approximation at frequency $\omega_{\mu}$.i.e., for each operator $O$, we have $\tilde{O}=O \exp \left(i \omega_{\mu} t\right)$ and we neglect the fast rotating terms,one gets

$$
\delta \dot{\tilde{c}}_{i}=-\frac{\kappa}{2} \delta \tilde{c}_{i}-G \delta \tilde{b}_{i}+\sqrt{\kappa} \tilde{c}_{i}^{i n} \quad, \quad \delta \dot{\tilde{b}}_{i}=-\frac{\gamma}{2} \delta \tilde{b}_{i}+G \delta \tilde{c}_{i}+\sqrt{\gamma} \tilde{\xi}_{i},
$$

Finally, using the Fourier transform of the last differential equations, the explicit expressions for $\delta \tilde{c}_{i}$ and $\delta \tilde{b}_{i}$ write

$$
\begin{aligned}
& \delta \tilde{c}_{i}(\omega)=\frac{-G}{d(\omega)} \sqrt{\gamma} \tilde{\xi}_{i}(\omega)+\frac{\left(\frac{\gamma}{2}+i \omega\right)}{d(\omega)} \sqrt{\kappa} \tilde{c}_{i}^{i n}(\omega), \\
& \delta \tilde{b}_{i}(\omega)=\frac{\left(\frac{\kappa}{2}+i \omega\right)}{d(\omega)} \sqrt{\gamma} \tilde{\xi}_{i}(\omega)+\frac{G}{d(\omega)} \sqrt{\kappa} \tilde{c}_{i}^{i n}(\omega),
\end{aligned}
$$

with $d(\omega)=G^{2}+\left(\frac{\gamma}{2}+i \omega\right)\left(\frac{\kappa}{2}+i \omega\right)$.

\subsection{Covariance matrix}

To estimate entanglement and Gaussian quantum discord between different bipartite modes selected from the global system, we will derive the explicit formula of the covariance matrix describing the whole system. For this, we introduce the following quadrature operators (EPR-type quadrature operators for mechanical and optical modes)

$$
\begin{array}{rlrl}
\delta X^{\mathrm{m}_{i}}(\omega) & =\frac{\delta \tilde{b}_{i}^{\dagger}+\delta \tilde{b}_{i}}{\sqrt{2}}, & \delta Y^{\mathrm{m}_{i}}(\omega)=i \frac{\delta \tilde{b}_{i}^{\dagger}-\delta \tilde{b}_{i}}{\sqrt{2}} \\
\delta X^{\mathrm{o}_{i}}(\omega)=\frac{\delta \tilde{c}_{i}^{\dagger}+\delta \tilde{c}_{i}}{\sqrt{2}}, & \delta Y^{\mathrm{o}_{i}}(\omega)=i \frac{\delta \tilde{c}_{i}^{\dagger}-\delta \tilde{c}_{i}}{\sqrt{2}}
\end{array}
$$

where $\delta X^{s_{i}}$ and $\delta Y^{s_{i}}$ are respectively the $i^{\text {th }}(i=1,2)$ position and momentum quadrature operators associated to the mechanical modes Eq. (17) (with $s \equiv \mathrm{m})$ and the optical modes Eq. (18) $(s \equiv$ o ). For 
continuous variables, it is appropriate to specify the system within the covariance matrix formalism [22, 39, 40]. We introduce the 8-component vector

$$
U^{\mathrm{T}}=\left(\delta X^{\mathrm{m}_{1}}(\omega), \delta X^{\mathrm{m}_{2}}(\omega), \delta Y^{\mathrm{m}_{1}}(\omega), \delta Y^{\mathrm{m}_{2}}(\omega), \delta X^{\mathrm{o}_{1}}(\omega), \delta X^{\mathrm{o}_{2}}(\omega), \delta Y^{\mathrm{o}_{1}}(\omega), \delta Y^{\mathrm{o}_{2}}(\omega)\right),
$$

where the subscript $\mathrm{T}$ stands for the transposition operation. The corresponding covariance matrix elements can be evaluated explicitly by using the correlations properties of the noise operators $c_{i}^{i n}$ and $\xi_{i}$ ( Eqs. (4)-(9)) and the following relation [28]

$$
\sigma_{p q}=\frac{1}{4 \pi^{2}} \int_{-\infty}^{+\infty} \int_{-\infty}^{+\infty} d \omega d \omega^{\prime} e^{-i\left(\omega+\omega^{\prime}\right) t} \sigma_{p q}\left(\omega, \omega^{\prime}\right)
$$

where the frequency-domain correlation function between the elements $p$ and $q$ of the vector $U^{\mathrm{T}}$ are defined by

$$
\sigma_{p q}\left(\omega, \omega^{\prime}\right)=\frac{1}{2}\left\langle\left\{U_{p}(\omega), U_{q}\left(\omega^{\prime}\right)\right\}\right\rangle,
$$

for $p, q=1, . ., 8$. After some algebra, we finally obtain

$$
\sigma=\left(\begin{array}{cccccccc}
a_{1} & 0 & c_{1} & 0 & c_{3} & 0 & c_{4} & 0 \\
0 & a_{1} & 0 & -c_{1} & 0 & c_{3} & 0 & -c_{4} \\
c_{1} & 0 & a_{1} & 0 & c_{4} & 0 & c_{3} & 0 \\
0 & -c_{1} & 0 & a_{1} & 0 & -c_{4} & 0 & c_{3} \\
c_{3} & 0 & c_{4} & 0 & a_{2} & 0 & c_{2} & 0 \\
0 & c_{3} & 0 & -c_{4} & 0 & a_{2} & 0 & -c_{2} \\
c_{4} & 0 & c_{3} & 0 & c_{2} & 0 & a_{2} & 0 \\
0 & -c_{4} & 0 & c_{3} & 0 & -c_{2} & 0 & a_{2}
\end{array}\right),
$$

where

$$
\begin{array}{rlrl}
a_{1} & =\frac{\beta \cosh 2 r}{2(1+\alpha)(1+\beta)}+\frac{\left(2 n_{\mathrm{th}}+1\right)(1+\alpha+\alpha \beta)}{2(1+\alpha)(1+\beta)} & c_{1} & =\frac{\beta \sinh 2 r}{2(1+\alpha)(1+\beta)}, \\
a_{2}=\frac{\cosh 2 r(1+\alpha+\beta)}{2(1+\alpha)(1+\beta)}+\frac{\left(2 n_{\mathrm{th}}+1\right) \alpha \beta}{2(1+\alpha)(1+\beta)} & c_{2}=\frac{\sinh 2 r(1+\alpha+\beta)}{2(1+\alpha)(1+\beta)} \\
c_{3}=\frac{\sqrt{\alpha \beta}}{2(1+\alpha)(1+\beta)}\left(-\left(2 n_{\mathrm{th}}+1\right)+\cosh 2 r\right) & c_{4}=\frac{\sqrt{\alpha \beta} \sinh 2 r}{2(1+\alpha)(1+\beta)},
\end{array}
$$

where $\alpha=\frac{\gamma}{\kappa}$ is the damping ratio [41] and $\beta=\frac{4 G^{2}}{\kappa \gamma}$ represents the opto-mechanical cooperativity. This parameter measures the coupling degree between mechanical and optical modes [17, 42].

\section{Entanglement analysis via the logarithmic negativity}

From the covariance matrix given by Eq. (21), we shall now investigate the bipartite entanglement between different modes in the system. Indeed, we quantify the quantum correlations using the logarithmic negativity between: the mechanical mode 1 and the mechanical mode 2 (subsystem (i)), the optical mode 1 and the optical mode 2 (subsystem (ii)), the mechanical mode 1 (resp. 2) and the 
optical mode 1 (resp. 2) (subsystem (iii)) and finally the mechanical mode 1 (resp. 2) and the optical mode 2 (resp. 1) (subsystem (iv)). For each pair of modes, the corresponding covariance matrix can be derived from the global covariance matrix (21). We have

$$
\begin{array}{ccc}
\sigma_{(\mathrm{i})}=\left(\begin{array}{cccc}
a_{1} & 0 & c_{1} & 0 \\
0 & a_{1} & 0 & -c_{1} \\
c_{1} & 0 & a_{1} & 0 \\
0 & -c_{1} & 0 & a_{1}
\end{array}\right) & \sigma_{(\mathrm{ii})}=\left(\begin{array}{cccc}
a_{2} & 0 & c_{2} & 0 \\
0 & a_{2} & 0 & -c_{2} \\
c_{2} & 0 & a_{2} & 0 \\
0 & -c_{2} & 0 & a_{2}
\end{array}\right), \\
\sigma_{(\mathrm{iii})}=\left(\begin{array}{cccc}
a_{1} & 0 & c_{3} & 0 \\
0 & a_{1} & 0 & c_{3} \\
c_{3} & 0 & a_{2} & 0 \\
0 & c_{3} & 0 & a_{2}
\end{array}\right) & \sigma_{(\mathrm{iv})}=\left(\begin{array}{cccc}
a_{1} & 0 & c_{4} & 0 \\
0 & a_{1} & 0 & -c_{4} \\
c_{4} & 0 & a_{2} & 0 \\
0 & -c_{4} & 0 & a_{2}
\end{array}\right) .
\end{array}
$$

The matrices (25] 26) are of the form

$$
\sigma_{(\mathrm{j})}=\left(\begin{array}{cc}
A_{(\mathrm{j})} & C_{(\mathrm{j})} \\
C_{(\mathrm{j})}^{T} & B_{(\mathrm{j})}
\end{array}\right),
$$

with $\mathrm{j} \in \operatorname{subsystems}\{(\mathrm{i})$, (ii), (iii), (iv) $\}$. The covariance matrix $\sigma_{(\mathrm{j})}$ is real, symmetric and positive and has block structure where $A_{(\mathrm{j})}, B_{(\mathrm{j})}$ and $C_{(\mathrm{j})}$ (and its transpose) are $2 \times 2$ Hermitian matrices. For each subsystem $\mathrm{j}, A_{(\mathrm{j})}$ and $B_{(\mathrm{j})}$ denote the symmetric covariance matrices for the individual reduced one-mode states and the matrix $C_{(\mathrm{j})}$ comprise the cross-correlations between modes. The logarithmic negativity is defined by [26, 27]

$$
E_{N}^{(\mathrm{j})}=\max \left\{0,-\ln \left(2 \eta_{(\mathrm{j})}^{-}\right)\right\}
$$

where $\eta_{(\mathrm{j})}^{-}$is the smallest simplistic eigenvalue of the partial transpose of the $4 \times 4$ covariance matrix $\sigma_{(\mathrm{j})}[27]$. It writes

$$
\eta_{(\mathrm{j})}^{-}=\sqrt{\frac{\tilde{\Delta}_{(\mathrm{j})}-\sqrt{\tilde{\Delta}_{(\mathrm{j})}^{2}-4 \operatorname{det} \sigma_{(\mathrm{j})}}}{2}},
$$

where the symbol $\tilde{\Delta}_{(\mathrm{j})}$ stands for the symplectic invariant for the covariance matrix (27). It is given by [27]

$$
\tilde{\Delta}_{(\mathrm{j})}=\operatorname{det} A_{(\mathrm{j})}+\operatorname{det} B_{(\mathrm{j})}-2 \operatorname{det} C_{(\mathrm{j})} .
$$

Using the results (22) and (23), one gets

$$
\begin{gathered}
2 \eta_{(\mathrm{i})}^{-}=2\left(a_{1}-c_{1}\right)=\frac{1+2 n_{\mathrm{th}}}{1+\alpha}\left(\frac{1}{1+\beta}+\alpha\right)+\frac{\beta e^{-2 r}}{(1+\alpha)(1+\beta)}, \\
2 \eta_{(\mathrm{ii})}^{-}=2\left(a_{2}-c_{2}\right)=\frac{1+2 n_{\mathrm{th}}}{1+\alpha} \frac{\alpha \beta}{1+\beta}+\frac{e^{-2 r}}{1+\alpha}\left(\frac{\alpha}{1+\beta}+1\right) .
\end{gathered}
$$

For the subsystems (iii) and (iv). The expressions of $2 \eta_{(\mathrm{iii})}^{-}$and $2 \eta_{(\mathrm{iv})}^{-}$are too cumbersome and will not be reported here. Clearly, the entanglement occurs when $E_{N}^{(\mathrm{j})}>0$ or equivalently $\eta_{(\mathrm{j})}^{-}<1 / 2$ with $\mathrm{j} \in$ subsystem $\{(\mathrm{i}),(\mathrm{ii}),(\mathrm{iii}),(\mathrm{iv})\}$. The simplistic eigenvalues $\eta_{(\mathrm{j})}^{-}$are a function of the squeezing parameter 
$r$, the opto-mechanical cooperativity $\beta$, the mean thermal photons number $n_{\text {th }}$ or equivalently the thermal bath temperature $T$ and the damping ratio $\alpha=\frac{\gamma}{\kappa}$.

As we shall hereafter focus on the difference between the logarithmic negativity and the Gaussian quantum discord as quantifiers of the quantum correlations, an appropriate choice of the parameters characterizing the system is needed. In other hand, this must corresponds to situations that can be implemented experimentally. In this respect, we consider some parameters reported in [43. The two cavities are characterized by the length $L=25 \mathrm{~mm}$, the laser wave length is $\lambda=1064 \mathrm{~nm}$, the frequency $\omega_{c}=2 \pi \times 5.26 \times 10^{14} \mathrm{~Hz}$ and pumped by a coherent laser source with power $P=11 \mathrm{~mW}$. The movable mirrors having the mass $\mu=145 \mathrm{ng}$ and oscillate at the frequency $\omega_{\mu}=2 \pi \times 947 \times 10^{3} \mathrm{~Hz}$ with the mechanical damping rate $\gamma=2 \pi \times 140 \mathrm{~Hz}$.
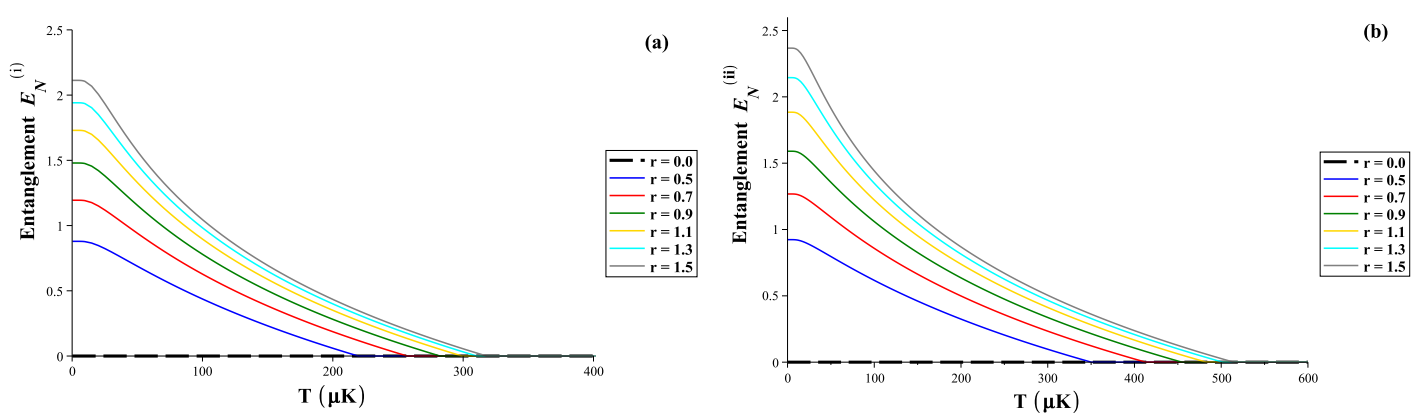

Figure 2: The logarithmic negativity $E_{N}$ versus the thermal bath temperature $T$ for various values of the squeezing parameter $r$. (a): the logarithmic negativity $E_{N}^{(\mathrm{i})}$ of the subsystem (i) formed by two identical mechanical modes, (b): the logarithmic negativity $E_{N}^{(i i)}$ of the subsystem (ii) composed by two identical optical modes. For both cases (a) and (b), the opto-mechanical cooperativity $\beta$ is taken equal to 34 , the damping ratio $\alpha=\frac{\gamma}{\kappa}$ is fixed to 0.05 (or equivalently $\kappa=2 \pi \times 2800 \mathrm{~Hz}$ ).

Fig.2 shows that the logarithmic negativity $E_{N}^{(\mathrm{i})}\left(\operatorname{resp} . E_{N}^{(\mathrm{ii})}\right)$ for the subsystems (i) (resp. (ii)) decreases when the thermal bath temperature $T$ increases. In particular, it is clearly seen that the logarithmic negativity $E_{N}^{(\mathrm{i})}$ vanishes more quickly than $E_{N}^{(\mathrm{ii})}$ under the temperature effects. We notice also that, for a fixed value of the thermal bath temperature, the quantities $E_{N}^{(\mathrm{i})}$ and $E_{N}^{(\mathrm{ii})}$ increase as the squeezing parameter increases. We remark that in the absence of the squeezed light $(r=0)$, the two mechanical modes of the subsystem (i) and the two optical modes of the subsystem (ii) remain separable (see the black dashed lines in Figs.2(a) and 2(b)). This reflects the relationship between the entanglement and the squeezed light explains the quantum correlations transfer from squeezed light to subsystems (i) and (ii) in agreement with the results obtained in [17]. From Fig.2 we also see that when the squeezing parameter $r$ increases, the critical value of the thermal bath temperature denoted $T_{0}$, from which the subsystems (i) and (ii) become separable decreases. The temperature $T_{0}$ is given by

$$
\frac{1}{T_{0}^{(\mathrm{i})}}=\frac{k_{B}}{\hbar \omega_{\mu}} \ln \left(\frac{2(1+\alpha+\alpha \beta)}{\beta\left(1-e^{-2 r}\right)}+1\right),
$$

for the subsystem (i) formed by the two mechanical modes. For the case of the optical modes (sub- 
system (ii)), it writes

$$
\frac{1}{T_{0}^{(\mathrm{ii})}}=\frac{k_{B}}{\hbar \omega_{\mu}} \ln \left(\frac{2 \alpha \beta}{(1+\alpha+\beta)\left(1-e^{-2 r}\right)}+1\right) .
$$

For the optical modes (the subsystem (ii)), the logarithmic negativity is more resilient against the temperature effects in comparison with the mechanical modes (the subsystem (i)). In fact, the logarithmic negativity is zero beyond $T=3 \times 10^{-4} \mathrm{~K}$ (for the mechanical modes) and $T=5 \times 10^{-4} \mathrm{~K}$ (for the optical modes) regardless the value of the squeezing parameter. Such a phenomenon is regularly known as entanglement sudden death (ESD) [44, 45]. In Fig.3 we give the evolution of the logarithmic
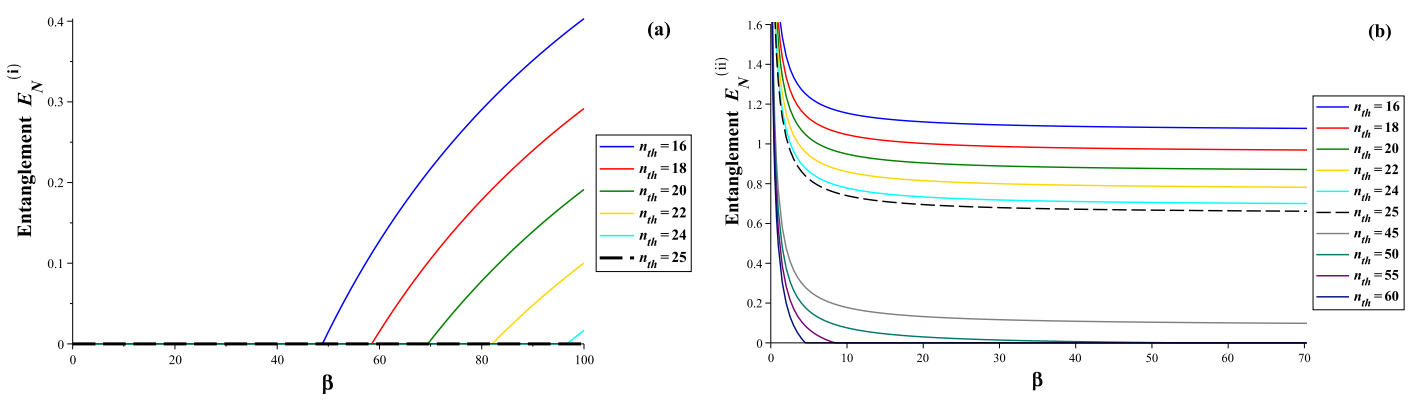

Figure 3: Plots of the logarithmic negativity $E_{N}$ versus the opto-mechanical cooperativity $\beta$ for various values of the mean thermal photons number $n_{\text {th }}$. (a): the logarithmic negativity $E_{N}^{(\mathrm{i})}$ of the subsystem (i) formed by two identical mechanical modes, (b): the logarithmic negativity $E_{N}^{(\text {ii) }}$ of the subsystem (ii) composed by two identical optical modes. In the two cases (a) and (b), we used $\alpha=\frac{\gamma}{\kappa}=0.01$ (or equivalently $\kappa=2 \pi \times 14 \times 10^{3} \mathrm{~Hz}$ ). The squeezing parameter $\mathrm{r}$ is fixed as $r=2$.

negativity $E_{N}^{(\mathrm{i})}$ and $E_{N}^{(\mathrm{ii})}$ versus the opto-mechanical cooperativity $\beta$ for various values of the mean thermal photons number $n_{\text {th }}$. The mechanical modes exhibit vanishing the logarithmic negativity for $n_{\mathrm{th}}>25$. For $n_{\mathrm{th}}<25$, when $\beta$ increases, the mechanical modes are entangled. The threshold value of the cooperativity $\beta_{0}^{(\mathrm{i})}$ beyond which the mechanical modes cease to be separable, is given by

$$
\beta_{0}^{(\mathrm{i})}=\frac{2 n_{\mathrm{th}}(1+\alpha)}{1-2 \alpha n_{\mathrm{th}}-e^{-2 r}} .
$$

The optical modes remain entangled for $n_{\text {th }}<25$ regardless the value taken by the cooperativity. However, for higher values of $n_{\mathrm{th}}$ ( $n_{\mathrm{th}}>25$ comparing with the case of the mechanical modes), they start to be separable above the critical value $\beta_{0}^{(i i)}$ given by

$$
\beta_{0}^{(\mathrm{ii})}=\frac{\left(e^{-2 r}-1\right)(1+\alpha)}{1-2 \alpha n_{\mathrm{th}}-e^{-2 r}} .
$$

Clearly, by increasing the mean thermal photons number $n_{\text {th }}$, the mechanical modes require a large value of $\beta$ to switch from separable states to entangled states (see Fig.2(a)). This behavior can be explained by the decoherence phenomenon. Indeed, $n_{\mathrm{th}}$ increases when the thermal bath temperature $T$ increases and therefore the environment effect on the system becomes more aggressive. Unlike the mechanical modes (see Fig.2(a)), $E_{N}^{(i i)}$ diminishes when $\beta$ increases (see Fig.2(b)). We now consider the case of the hybrid opto-mechanical systems, formed by an optical cavity mode and a mechanical mode. We start analyzing the hybrid subsystem (iii) which is composed by two interacting modes (an 
optical cavity mode and its corresponding mechanical mode). In this case, we have $\operatorname{det} C_{(\mathrm{iii})}=\left(c_{3}\right)^{2}>0$ and subsequently, according to [25, 46] ( $\operatorname{det} C<0$ is a necessary condition for a two-mode Gaussian state to be entangled), the states of the two modes forming the hybrid subsystem (iii) are always separable. Different entanglement behavior is obtained for the hybrid subsystem (iv). The results are

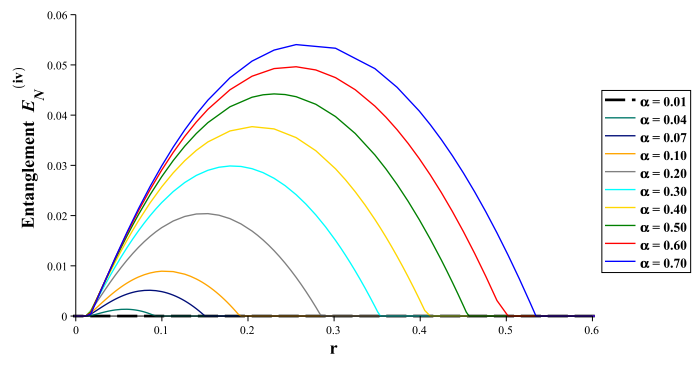

Figure 4: The logarithmic negativity $E_{N}^{(\mathrm{iv})}$ of the hybrid subsystem (iv) formed by two uncoupled modes (an optical cavity mode and non corresponding mechanical mode) versus the squeezing parameter $\mathrm{r}$ for different values of the damping ratio $\alpha$. The mean thermal photons number $n_{\mathrm{th}}$ and the opto-mechanical cooperativity $\beta$ are taken equal to 0.01 and 1 respectively.

reported in Fig.4. The logarithmic negativity $E_{N}^{(\mathrm{iv})}$ is depicted as a function of the squeezing parameter $\mathrm{r}$ for various values of the damping ratio $\alpha$. In the absence and also for small values of the squeezing parameter $r$, Fig.4 reveals that no entanglement between the two modes forming the subsystem (iv). This indicates that the squeezed light is a necessary element to achieve entanglement. Such a result traduces the transfer of the quantum correlations from the squeezed light to the subsystem (iv), which agrees with the results obtained in Figs.2(a) and 2(b). Fig.4 shows a resonant behavior of the entanglement $E_{N}^{(\mathrm{iv})}$ in term of the squeezing parameter $r$. The maximum value of $E_{N}^{(\mathrm{iv})}$ increases with increasing values of the damping ratio $\alpha$. It must be noticed that for a fixed value of $\alpha$, the entanglement $E_{N}^{(\mathrm{iv})}$ is enhanced when $r$ increases before the resonance. This is no longer valid after passing the resonant value of $E_{N}^{(\mathrm{iv})}$. Indeed, for higher values of $r$, the entanglement goes to zero. This is mainly due to thermal noise (affected each cavity) enhanced by strong squeezing light as obtained in $[28]$.

\section{Gaussian quantum discord}

In this section, we shall investigate the usefulness of the Gaussian quantum discord [21, 22] in comparison with the logarithmic negativity discussed in the previous section. In particular, we shall focus on the situations, discussed in section III, where the logarithmic negativity is zero. Hence, using the Gaussian quantum discord defined in [21, 22], we evaluate the quantum correlations present in different subsystems (i), (ii), (iii) and (iv) at the separable states. For the bipartite subsystem j $(\mathrm{j} \in \operatorname{subsystems}\{(\mathrm{i}),(\mathrm{ii}),(\mathrm{iii}),(\mathrm{iv})\})$ described by the covariance matrix $\sigma_{(\mathrm{j})}$ (Eq. (27)), the Gaussian quantum discord is given by [21, 22$]$

$$
D^{(\mathrm{j})}=f\left(\sqrt{\operatorname{det} B_{(\mathrm{j})}}\right)-f\left(\nu_{+}^{(\mathrm{j})}\right)-f\left(\nu_{-}^{(\mathrm{j})}\right)+f\left(\varepsilon^{(\mathrm{j})}\right),
$$


where the function $f$ is defined by $f(x)=\left(x+\frac{1}{2}\right) \log _{2}\left(x+\frac{1}{2}\right)-\left(x-\frac{1}{2}\right) \log _{2}\left(x-\frac{1}{2}\right)$. The symplectic eigenvalues $\nu_{+}^{(\mathrm{j})}$ and $\nu_{-}^{(\mathrm{j})}$ are defined by [21, 22]

$$
\nu_{ \pm}^{(\mathrm{j})}=\sqrt{\frac{\Delta_{(\mathrm{j})} \pm \sqrt{\Delta_{(\mathrm{j})}^{2}-4 \operatorname{det} \sigma_{(\mathrm{j})}}}{2}}
$$

with $\Delta_{(\mathrm{j})}=\operatorname{det} A_{(\mathrm{j})}+\operatorname{det} B_{(\mathrm{j})}+2 \operatorname{det} C_{(\mathrm{j})}$. For the bipartite subsystems (i), (ii) and (iv) described respectively by the covariance matrices $\sigma_{(\mathrm{i})}, \sigma_{(\mathrm{ii})}$ and $\sigma_{(\mathrm{iv})}, \varepsilon^{(\mathrm{j})}$ takes the following form [21]

$$
\varepsilon^{(\mathrm{j})}=\frac{\sqrt{\operatorname{det} A_{(\mathrm{j})}}+2 \sqrt{\operatorname{det} A_{(\mathrm{j})} \operatorname{det} B_{(\mathrm{j})}}+2 \operatorname{det} C_{(\mathrm{j})}}{1+2 \sqrt{\operatorname{det} B_{(\mathrm{j})}}},
$$

with $\mathrm{j} \in \operatorname{subsystem}\{(\mathrm{i}),(\mathrm{ii}),(\mathrm{iv})\}$. For the subsystem (iii) defined by the matrix $\sigma_{(\mathrm{iii})}$, we have $C_{(\mathrm{iii})}=\operatorname{diag}\left(c_{3},+c_{3}\right)$. Then $\varepsilon^{(\mathrm{j})}$ is given by the formula [22, 23 ]

$$
\varepsilon^{(\mathrm{j})}=\frac{2\left|\operatorname{det} C_{(\mathrm{j})}\right|+\sqrt{4\left(\operatorname{det} C_{(\mathrm{j})}\right)^{2}+\left(4 \operatorname{det} B_{(\mathrm{j})}-1\right)\left(4 \operatorname{det} \sigma_{(\mathrm{j})}-\operatorname{det} A_{(\mathrm{j})}\right)}}{\left(4 \operatorname{det} B_{(\mathrm{j})}-1\right)},
$$

with $\mathrm{j} \equiv$ (iii). Having the necessary ingredients to deal with the Gaussian quantum discord for the
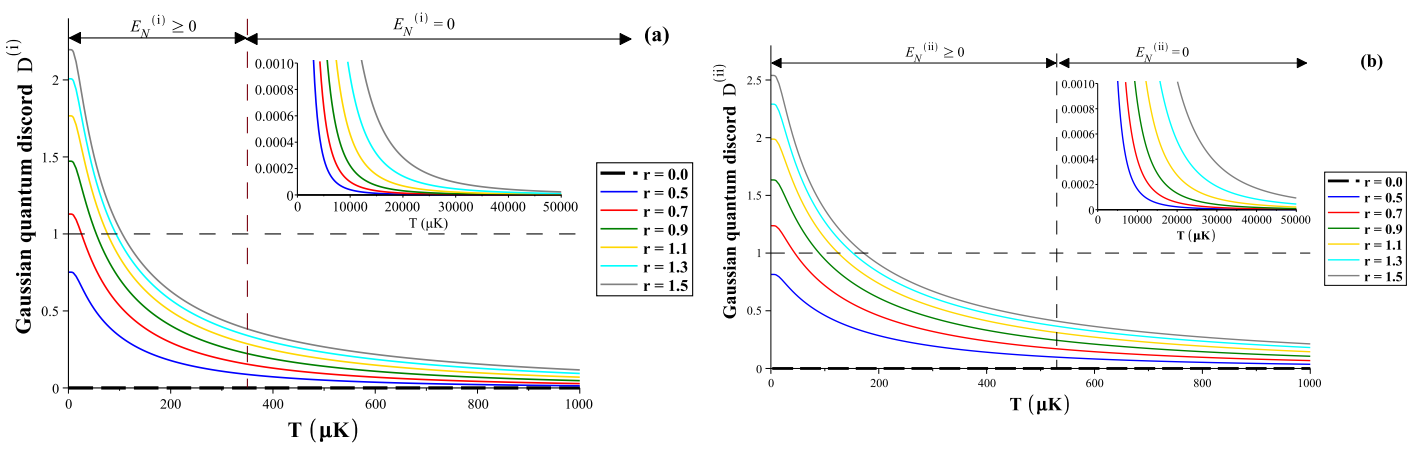

Figure 5: Plots of the Gaussian quantum discord $D$ against the thermal bath temperature $T$ for various values of the squeezing parameter $r$. (a): the Gaussian quantum discord $D^{(\mathrm{i})}$ of the subsystem (i), (b): the Gaussian quantum discord $D^{(\mathrm{ii})}$ of the subsystem (ii). For both cases (a) and (b), the parameters $\alpha=\frac{\gamma}{\kappa}$ and $\beta$ are fixed as the same as in Fig.2. The vertical dashed lines show the boundary between the separable states $\left(E_{N}=0\right)$ and entangled states $\left(E_{N} \neq 0\right)$. Figs. $(5 \mathrm{a})$ and $(5 \mathrm{~b})$ show that the Gaussian quantum discord presents non-zero values in the same regions where the subsystems (i) and (ii) are separable (see Figs.2(a) and 2(b)), which is an indicator of quantumness of correlations in the considered subsystems (existence of non-classical correlations even at the separable states).

various subsystems of the opto-mechanical system under consideration, we investigate firstly the quantum correlations measured by $D^{(\mathrm{i})}$ and $D^{(\mathrm{ii})}$ present respectively in the homogeneous subsystems (i) and (ii). In Fig.5 we give the variations of $D^{(\mathrm{i})}$ and $D^{(\mathrm{ii})}$ as function of the thermal bath temperature $T$ for different values of the squeezing parameter $r$. The damping ratio $\alpha$ and the opto-mechanical cooperativity $\beta$ take the same values as in Fig.2. Clearly, the quantum discord $D^{(\mathrm{i})}$ (for the mechanical modes) and $D^{(i i)}$ (for the optical modes) decrease when the thermal bath temperature increases. 
Using the results reported in Figs. $(2 \mathrm{a})$ and $(2 \mathrm{~b})$ we notice that the logarithmic negativity vanishes beyond $T \approx 3.5 \times 10^{-4} K$ for the mechanical modes $\left(E_{N}^{(\mathrm{i})}=0\right)$ and beyond $T \approx 4.75 \times 10^{-4} K$ for the optical modes $\left(E_{N}^{(\mathrm{ii})}=0\right)$. However, for the already mentioned ranges of thermal bath temperature, the Gaussian quantum discord $D^{(\mathrm{i})}$ and $D^{(\mathrm{ii})}$ are non zero. This indicates that the Gaussian quantum discord measure seems more robust and resilient versus the effect of the environment (decoherence) and constitutes a good tool to decide about the existence of non-classical correlations (quantumness) in opto-mechanical systems. This result corroborate the fact that quantum correlations exist in the subsystems (i) and (ii) even at the separable states. We note also that, when $E_{N}^{(\mathrm{j})}=0$ we have $D^{(\mathrm{j})}<1$ with $\mathrm{j} \in \operatorname{subsystem}\{(\mathrm{i}),(\mathrm{ii})\}$, which is agrees with the analysis reported in [21, 22]. Another important aspect, we investigate in this paper, concerns the behavior of the Gaussian quantum discord of the mechanical and optical modes (the subsystem (i) and (ii)) in terms of the opto-mechanical cooperativity $\beta$. This is reported in Fig.6. The Gaussian quantum discord is plotted as a function of the opto-mechanical cooperativity $\beta$ for various values of the mean thermal photons number $n_{\text {th }}$. The damping ratio $\alpha$ and the squeezing parameter $r$ are fixed as in Fig.3 in order to compare the logarithmic negativity with the Gaussian quantum discord as quantifiers of quantum correlations in the subsystems (i) and (ii). The Gaussian quantum discord $D^{(\mathrm{i})}$ increases with increasing values of
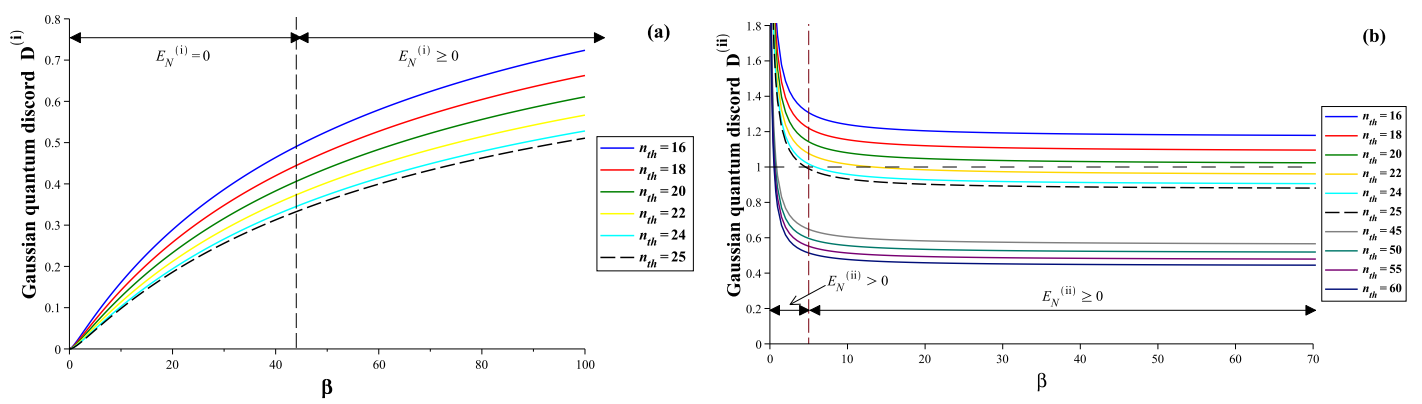

Figure 6: The Gaussian quantum discord $D$ versus the opto-mechanical cooperativity $\beta$ for various values of the mean thermal photons number $n_{\text {th. }}$. Panel (a) shows the Gaussian quantum discord $D^{(\mathrm{i})}$ of the subsystem (i), panel (b) shows the Gaussian quantum discord $D^{(i i)}$ of the subsystem (ii). The parameters $\alpha$ and $r$ are fixed as the same as in Fig.3. The vertical dashed lines show the boundary between, separable and entangled states. It is easy to remark that: for the subsystem (i), when $\beta \in[0,50], E_{N}^{(\mathrm{i})}=0$ and $D^{(\mathrm{i})} \neq 0$ (see Figs.(3a) and (6a)), concerning the subsystem (ii) and focusing on the case where $n_{\mathrm{th}}=60$, we can see that, for $\beta>5, E_{N}^{(\mathrm{ii})}=0$ and $D^{(\mathrm{ii})} \neq 0$ (see Figs.3(b) and $6(\mathrm{~b}))$. Therefore, such situations, make sure the existence of quantumness of correlations between the two modes formed the subsystems (i) and (ii).

the cooperativity $\beta$ (see Fig.6(a)) but this increasing becomes slow for higher mean thermal photons number $n_{\text {th }}$. In the other hand, the quantum discord $D^{(\mathrm{ii})}$ decreases as the cooperativity increases and becomes almost constant for higher values of $\beta$. It must be also noticed that for the non separable optical modes, the diminution of the quantum discord is more pronounced for higher thermal photons number $n_{\text {th }}$. Comparing Figs.6(a) and 6(b) we deduce that there is a tradeoff of the intricacy between the optical and mechanical modes. Indeed, for small values of the cooperativity $\beta$, the mechanical 
modes are separable while the optical modes are not and by increasing the cooperativity, the mechanical modes become non separable and the optical modes are separable. Now, we consider the Gaussian
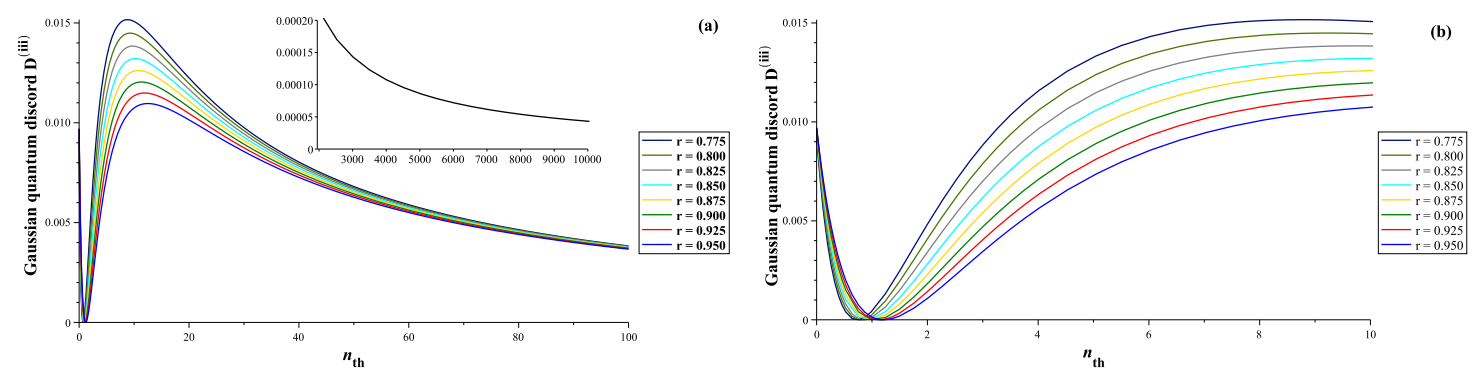

Figure 7: The Gaussian quantum discord $D^{(\mathrm{iii})}$ of the hybrid subsystem (iii) formed by two interacting modes (an optical cavity mode and its corresponding mechanical mode) versus the mean thermal photons number $n_{\mathrm{th}}$ ((a) behavior for high values of $n_{\mathrm{th}}$, (b) behavior for small values of $\left.n_{\mathrm{th}}\right)$ for different values of the squeezing parameter $r$. The damping ratio $\alpha$ and the opto-mechanical cooperativity $\beta$ are taken equal to 0.5 and 10 respectively.

quantum discord in the hybrid subsystem (iii). The robustness of the Gaussian quantum discord $D^{(\mathrm{iii})}$ with respect to the mean thermal photons number $n_{\text {th }}$ (or equivalently the thermal bath temperature $T$ ) for various values of the squeezing parameter $r$ is shown in Fig.7. This figure shows that $D^{(i i i)}$ has two distinct behaviors according to $n_{\mathrm{th}}$. Indeed, for small values of $n_{\mathrm{th}}\left(0<n_{\mathrm{th}}<1\right)$ and for a given value of $r, D^{(i i i)}$ decreases quickly from a non zero initial value, reaching a minimum around $n_{\mathrm{th}} \approx 1$ (see Fig.7(b)), whereas for $n_{\mathrm{th}}>1, D^{(\mathrm{iii})}$ has a resonant behavior (the maximums decrease when $r$ increase and attained around $n_{\mathrm{th}} \approx 10$ (see Fig.7(a)). Finally, it is clear that $D^{\text {(iii) }}$ remains non zero for high values of $n_{\mathrm{th}}\left(n_{\mathrm{th}}>10^{4}\right)$ and keeps a value almost constant independently of $r$ (see Fig.7(a)). We recall that the subsystem (iii) is always separable and the Gaussian quantum discord $D^{\text {(iii) }}$ is less than 1 . This is in agreement with the general properties of Gaussian quantum discord [21, 22]. Therefore the quantum correlations detected in this situation are a witness of quantumness. The behavior of the Gaussian quantum discord $D^{(\mathrm{iv})}$ of the hybrid subsystem (iv) is plotted as a

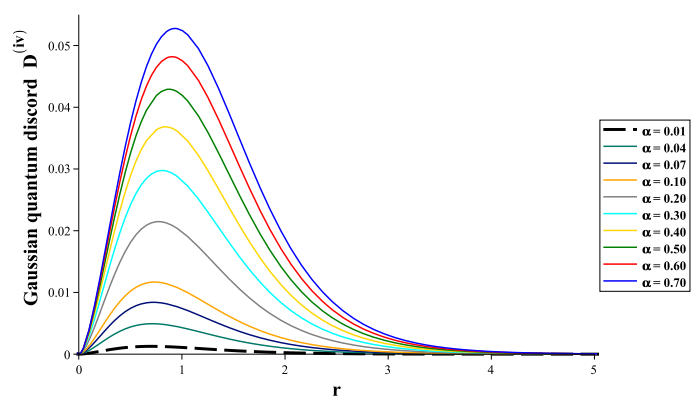

Figure 8: The Gaussian quantum discord $D^{(\mathrm{iv})}$ of the hybrid subsystem (iv) formed by an optical cavity mode and non corresponding mechanical mode versus the squeezing parameter $\mathrm{r}$ for different values of the damping ratio $\alpha$. The mean thermal photons number $n_{\text {th }}$ and the opto-mechanical cooperativity $\beta$ are taken equal to 0.01 and 1 respectively. 
function of the squeezing parameter $r$ in Fig.8, various values of the damping ratio $\alpha$ are considered. Fig. 8 shows that the Gaussian quantum discord is non zero for $0<r \leq 0.02$ and $r>0.55$ where the logarithmic negativity is zero (see Fig.4), in this case, we have also $D^{(\text {iv) }}<1$. Finally, using the standard homodyne detection method, it is possible to determine numerically the global covariance matrix ( Eq. (21) ) by the measure of the correlations between the output fields, which provides an experimental method to quantify stationary entanglement and Gaussian quantum discord by means of Eqs. (36) and (28). More technical details are presented in Refs. [10, 46],

\section{Concluding Remarks}

To summarize, we have investigated the quantum correlations in a quantum opto-mechanical system describing the interaction between light and mechanical systems in a Markovian environment without the adiabatic approximation. We considered an opto-mechanical system consisting by two identical Fabry-Perot cavities. We gave the quantum Langevin equations (see Eqs. (2) and (3)) from which we derived the dynamics of the optical as well as the mechanical degrees of freedom. A crucial feature is that all the quadratures of optical and mechanical modes are expanded to the first-order around the steady states (see Eqs. (10) and (11)). In this picture, the quantum Langevin equation gives a coupled system of differential equations involving noise operators (see Eqs. (12) and (13)). Our analysis is not very different from other proposals discussed recently in the literature. Differences become relevant when we incorporate in the model the quantum correlations in various bipartite subsystems (four subsystems). Indeed, given an arbitrary steady state, the fluctuations about it are fully characterized by its $8 \times 8$ covariance matrix of all pairwise correlations among the quadratures. To compute pairwise correlations, we used the $4 \times 4$ sub-matrices given by Eqs. ((25) $),(26))$ which are extracted from the global covariance matrix $\sigma$ (see Eq. (21)). They correspond to the four subsystems (i), (ii), (iii) and (iv). The covariance sub-matrix $\sigma_{(\mathrm{i})}\left(\right.$ resp. $\sigma_{(\mathrm{ii})}$ ) (see Eq. (25)) associated to the homogeneous subsystem (i) (resp. (ii)) describes the correlations between the mechanical (resp. optical) modes. On the other hand, the covariance sub-matrices $\sigma_{(\mathrm{iii})}$ and $\sigma_{(\mathrm{iv})}$ (see Eq. (26) ) associated with the hybrid subsystem (iii) and (iv)) contain the information about the quantum correlations between the mechanical and optical modes in the opto-mechanical system under consideration. This global description allows us to access to the non-classical correlations existing between each pair of the quadrature components. In evaluating the pairwise correlations, we deliberately considered the logarithmic negativity which characterizes the degree of entanglement and the Gaussian quantum discord which quantifies the non-classical correlations not captured by entanglement. A particular focus was devoted to states with vanishing logarithmic negativity (separable states) for which the Gaussian discord is non zero. We have depicted the opto-mechanical entanglement evolution under the thermal bath temperature, the opto-mechanical cooperativity, the squeezing parameter of the light and the mean thermal photons number. The results, reported in Figs.2(a) and 2(b), show that the entanglement between the optical modes (see Fig.2(b)) are more robust against the temperature 
effects than the mechanical modes (see Fig.2(a)). Furthermore, from Figs.2(a) and 2(b), it is clear that the squeezed light enhances the entanglement between the optical modes (subsystem (ii)) and the mechanical modes (subsystem (i)) especially for lower thermal bath temperatures. In the subsystem

(iv), the logarithmic negativity is quadratic in term of the squeezing parameter (i.e. $E_{N}^{(\text {iv) }} \sim r^{2}$ ). This indicates that to attain the maximal value of the correlations between the optical and mechanical modes in the subsystem (iv), one has to choose a special value of the squeezing parameter. The Gaussian quantum discord in the subsystem (i) and (ii) follows rigourously the same behavior in terms of the temperature. It is important to notice that for the subsystems (i), (ii) and (iv), which are formed by two spatially separable modes, it is indispensable to use the squeezed light to create entanglement and Gaussian quantum discord. This indicates the quantum correlations transfer from the squeezed light to the two considered modes. In the subsystem (iii), the mechanical mode and the optical mode are always separable (the logarithmic negativity is zero) but the corresponding pairwise quantum correlation is non zero when measured by Gaussian quantum discord. More interesting, for moderates values of mean thermal photons number $\mathrm{n}_{t h}$, the Gaussian quantum discord tends to an asymptotic constant value. This constitutes a very interesting and at the same time surprising result. Indeed, in the subsystem (iii), it seems that low thermal effect enhances the quantum correlations. Our results confirm the robustness of the Gaussian quantum discord, in comparison with the entanglement, for the four partitions (i), (ii), (iii) and (iv) comprised in the opto-mechanical system investigated in this paper.

\section{References}

[1] L. Tian and H. Wang, Phys. Rev. A 82 (2010) 053806.

[2] Y.D. Wang and A.A. Clerk, Phys. Rev. Lett. 108 (2012) 153603.

[3] S. Singh, H. Jing, E. M. Wright and P. Meystre, Phys. Rev. A 86 (2012) 021801.

[4] T.A. Palomaki, J.W. Harlow, J.D. Teufel, R.W. Simmonds and K.W. Lehnert, Nature 495 (2013) 210.

[5] S. Gigan, H. Bohm, M. Paternostro, F. Blaser, G. Langer, J. Hertzberg, K. Schwab, D. Bauerle, M. Aspelmeyer, and A. Zeilinger, Nature. 444 (2006) 67.

[6] D. Kleckner and D. Bouwmeester, Nature. 444 (2006) 75.

[7] S. Mancini, V. Giovannetti, D. Vitali and P. Tombesi, Phys. Rev. Lett. 88 (2002) 120401.

[8] J. Zhang, K. Peng and S.L. Braunstein, Phys. Rev. A 68 (2003) 013808.

[9] M. Pinard, A. Dantan, D. Vitali, O. Arcizet, T. Briant, A. Heidmann, Europhysics. Letters. 7 (2005) 747. 
[10] D. Vitali, S. Gigan, A. Ferreira, H.R. Bohm, P. Tombesi, A. Guerreiro, V. Vedral, A. Zeilinger and M. Aspelmeyer, Phys. Rev. Lett. 98 (2007) 030405.

[11] R. Ghobadi, A.R. Bahrampour and C. Simon, Phys. Rev. A 84 (2011) 063827.

[12] B. Rogers, M. Paternostro, G.M. Palma and G.D. Chiara, Phys. Rev. A 86 (2012) 042323.

[13] A. Mari and J. Eisert, New J. Phys 14 (2012) 075014.

[14] M.J. Hartmann and M.B. Plenio, Phys. Rev. Lett. 101 (2008) 200503.

[15] M. Paternostro, D. Vitali, S. Gigan, M.S. Kim, C. Brukner, J. Eisert and M. Aspelmeyer, Phys. Rev. Lett. 99 (2007) 250401.

[16] T. Huan, R. Zhou and H. Ian, arXiv:1502.04863 [quant-ph] (2015).

[17] E.A. Sete, H. Eleuch and C.H.R. Ooi, J. Opt. Soc. Am. B 31 (2014) 2821.

[18] W.K. Wootters, Phys. Rev. Lett. 80 (1998) 2245.

[19] H. Ollivier and W. H. Zurek, Phys. Rev. Lett. 88 (2001) 017901.

[20] L. Henderson and V. Vedral, J. Phys. A 34 (2001) 6899.

[21] P. Giorda and M.G.A. Paris, Phys. Rev. Lett. 105 (2010) 020503.

[22] G. Adesso and A. Datta, Phys. Rev. Lett. 105 (2010) 030501.

[23] S. Olivares, Eur. Phys. J. Special Topics 203 (2012) 3.

[24] L.M. Duan, G. Giedke, J.I. Cirac and P. Zoller, Phys. Rev.Lett. 84 (2000) 2722.

[25] R. Simon, Phys. Rev. Lett. 84 (2000) 2726.

[26] G. Vidal and R.F. Werner, Phys. Rev. A 65 (2002) 032314.

[27] G. Adesso et al, Phys. Rev. A 70 (2004) 022318.

[28] L. Mazzola and M. Paternostro, Phys. Rev. A 83 (2011) 062335.

[29] D.C. Burnham and D.L. Weinberg, Phys. Rev. Lett. 25 (1970) 84.

[30] Y.H. Shih and C.O. Alley, Phys. Rev. Lett. 61 (1988) 2921.

[31] R.E. Slusher, L. Hollberg, B. Yurke and J. Mertz, Phys. Rev. Lett. 55 (1985) 2409.

[32] M. Shahriar and P.R. Hemmer, Optics Communications. 158 (1998) 273.

[33] C.H. Metzger and K. Karrai, Nature. 432 (2004) 1002. 
[34] C.K. Law, Phys. Rev. A 51 (1995) 2537.

[35] S. Huang and G.S. Agarwal, New J. Phys 11 (2009) 103044.

[36] C.W. Gardiner, Phys. Rev. Lett. 56 (1986) 1917.

[37] V. Giovannetti and D. Vitali, Phys. Rev. A 63 (2001) 023812.

[38] C. Fabre, M. Pinard, S. Bourzeix, A. Heidmann, E. Giacobino and S. Reynaud, Phys. Rev. A 49 (1994) 1337.

[39] G. Adesso, S. Ragy and A.R. Lee, Open Syst. Inf. Dyn. 21 (2014) 1440001.

[40] S.L. Braunstein and P.V. Loock, Rev. Mod. Phys. 77 (2005) 513.

[41] Y.D. Wang and A.A. Clerk, Phys. Rev. Lett. 110 (2013) 253601.

[42] T.P. Purdy, P.L. Yu, R.W. Peterson, N.S. Kampel and C.A. Regal, Phys. Rev. X 3 (2013) 031012 .

[43] S. Gröblacher, K. Hammerer, M.R. Vanner and M.Aspelmeyer, Nature. 460 (2009) 724.

[44] M. Daoud and R.A. Laamara, Phys. Lett. A 376 (2012) 2361.

[45] A. AlQasimi and D.F.V. James, Phys. Rev. A 77 (2008) 012117.

[46] J. Laurat, G. Keller, J.A. Oliveira-Huguenin, C. Fabre, T. Coudreau, A. Serafini, G. Adesso and F. Illuminati, J. Opt. B: Quantum Semiclass.Opt. 7 (2005) S577. 